\title{
Quality indicators for colonoscopy: The road forward
}

\author{
David Armstrong MA MB BChir FRCPUK FACG AGAF FRCPC
}

$\mathrm{T}$ he widespread introduction of colon cancer screening programs over the past decade has highlighted the role of colonoscopy, both as an adjunct to population-based stool testing programs and as a primary screening tool. This has led to a dramatic increase in the number of colonoscopies performed and greater recognition of the need to ensure that this complex diagnostic and therapeutic procedure is performed to a consistently high standard. However, although colonoscopy was first introduced $>5$ decades ago, it is only relatively recently that there have been any systematic approaches to quality assurance and quality improvement in endoscopy. In this context, the systematic review and clinical practice guideline on colonoscopy quality assurance in Ontario, published in the current issue of the Journal (1) (pages 251-274), provides a welcome update to the 2007 Cancer Care Ontario colonoscopy standards (2). Although the article's focus is the quality of colonoscopy performed in Ontario, particularly with respect to colorectal cancer screening, the topics of physician training and competence, institutional quality assurance parameters and procedural quality indicators are highly relevant to other jurisdictions and to endoscopy performed for other indications.

Quality assurance and improvement in any field are predicated on the availability of outcomes data; in the absence of reliable data on current performance, it is impossible to benchmark or improve performance. The first step in quality assurance is, therefore, to identify appropriate indicators that will be recorded or measured; it is unrealistic to expect that there will be one universal quality metric, or that all ideal quality metrics can be identified or measured from the outset.

The quality indicators identified by Tinmouth et al (1) are an important first step in developing quality assurance programs for colonoscopy; some indicators, such as colonoscope withdrawal time, may be less important than previously believed while others, such as adenoma detection rate, are currently technically difficult to document. The key message, however, is that there are now evidence-based indicators and auditable outcomes that can be measured for colonoscopy quality assurance.

Quality improvement for colonoscopy and endoscopy, in general, should now progress to the rapid adoption of programs that can make use of these quality indicators. In Canada, provincial ministries of health and colorectal cancer screening programs, as well as health care institutions and endoscopy facilities, should be strongly encouraged to implement standardized electronic endoscopy reporting systems to permit reliable, accurate data collection (3) for feedback to endoscopy facilities and endoscopists so that they may implement targeted quality improvement programs. In the absence of installed electronic endoscopy reporting systems, point-of-care data collection using, for example, the Canadian Association of Gastroenterology PAGE practice audit program (4) also provides performance and quality indicator data, both for practising colonoscopists and for endoscopists in training (5).

As Tinmouth et al (1) have noted, there are also institutional elements in the delivery of high-quality colonoscopy; some less frequent quality indicators can be monitored only at an institution or regional level. More importantly, institutions and endoscopy facilities have a responsibility to foster a culture of measurement and quality improvement. Quality improvement tools, such as the Global Rating Scale Canada, enable endoscopy facilities to track key, patient-centred indicators including wait-times, comfort and safety in a standardized framework with access to shared resources to facilitate quality improvement (6). In particular, institutional quality programs should provide a mechanism using, for example, direct observation of procedural skills (7) to identify endoscopists who do not meet performance criteria. The availability of standardized quality indicator metrics provides endoscopists with clear criteria for performance evaluation and remediation, and supports the continued development of skills enhancement programs taught by trained faculty (8) to improve colonoscopy quality in a nonadversarial environment.

Further much-needed progress in providing high-quality endoscopy to meet the needs and expectations of Canadian patients requires a rapid move forward from the identification of key quality indicators (1) to the widespread adoption of comprehensive, integrated quality improvement programs that encompass repeated cycles of measurement, evaluation and intervention (6).

\section{REFERENCES}

1. Tinmouth J, Kennedy EB, Baron D, et al. Colonoscopy quality assurance in Ontario: Systematic review and clinical practice guideline. Can J Gastroenterol Hepatol 2014;28:251-74.

2. Rabeneck L, Rumble RB, Axler J, et al. Cancer Care Ontario Colonoscopy Standards. Toronto: Cancer Care Ontario; October 7, 2007. Program in Evidence-Based Care Evidence-Based Series No.:15-5.

3. Armstrong D, Barkun A, Bridges R, et al. Canadian Association of Gastroenterology consensus guidelines on safety and quality indicators in endoscopy. Can J Gastroenterol 2012;26:17-31.

4. Armstrong D, Hollingworth R, MacIntosh D, et al. Point-of-care, peer-comparator colonoscopy practice audit: The Canadian Association of Gastroenterology Quality Program - Endoscopy. Can J Gastroenterol 2011;25:13-20.

5. Xenodemetropoulos T, Armstrong D, Tse F, et al. Resident practice audit in gastroenterology (RPAGE): An innovative approach to gastroenterology trainee evaluation and professional development. Can J Gastroenterol 2013;27(Suppl A):A069. (Abst)

6. MacIntosh D, Dubé C, Hollingworth R, Veldhuyzen van Zanten S, Daniels S, Ghattas G. The endoscopy Global Rating Scale Canada: Development and implementation of a quality improvement tool. Can J Gastroenterol 2013;27:74-82.

7. Barton JR, Corbett S, van der Vleuten CP, English Bowel Cancer Screening Programme; UK Joint Advisory Group for Gastrointestinal Endoscopy. The validity and reliability of a Direct Observation of Procedural Skills assessment tool: Assessing colonoscopic skills of senior endoscopists. Gastrointest Endosc 2012;75:591-7

8. Skills Enhancement of Endoscopy. <www.cag-acg.org/skillsenhancement-for-endoscopy> (Accessed April 14, 2014). 


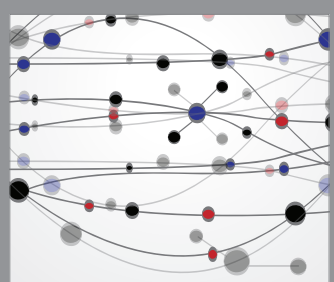

The Scientific World Journal
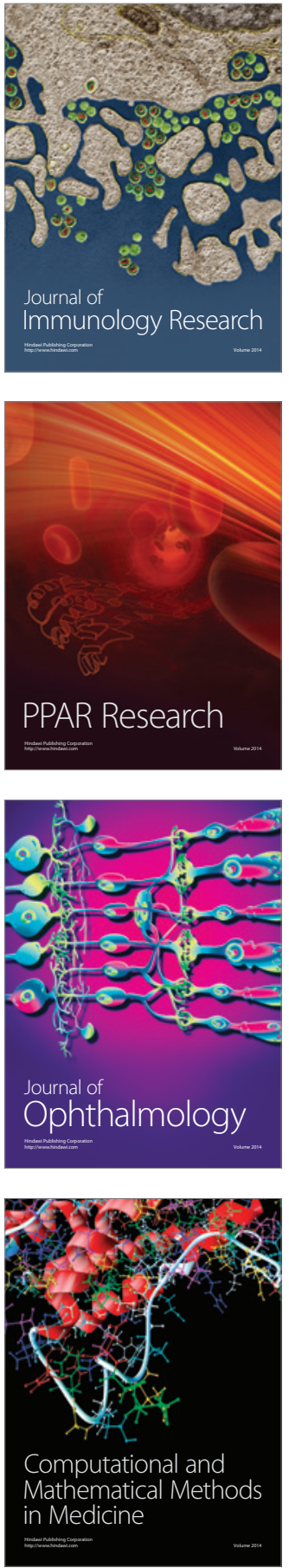

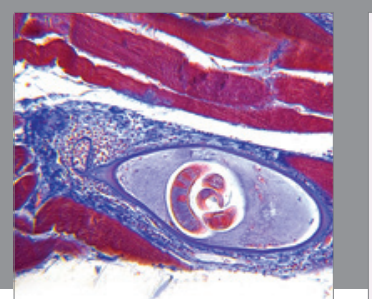

Gastroenterology Research and Practice

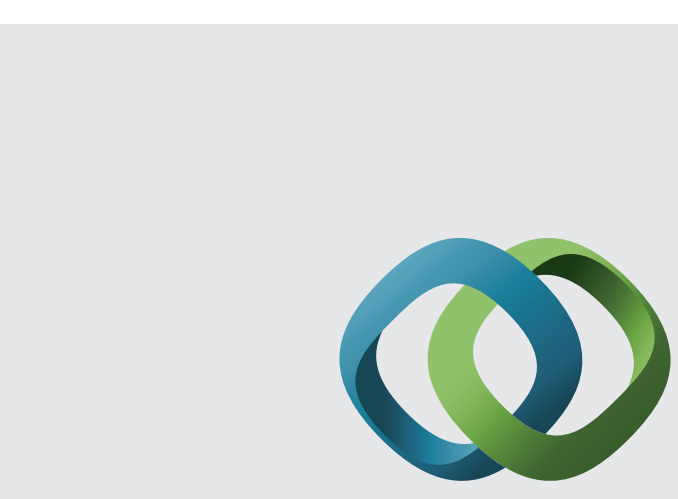

\section{Hindawi}

Submit your manuscripts at

http://www.hindawi.com
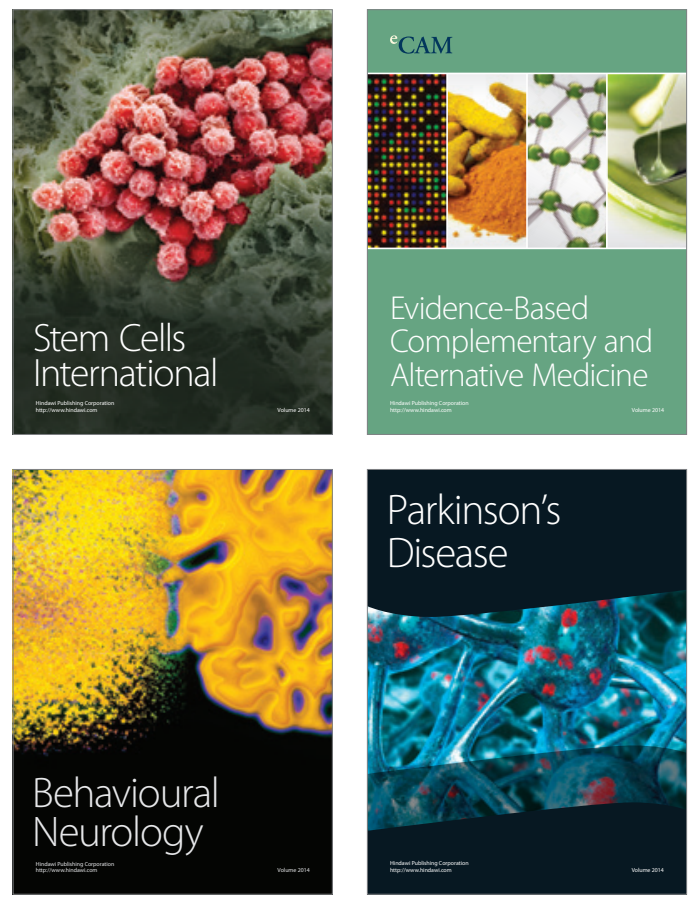
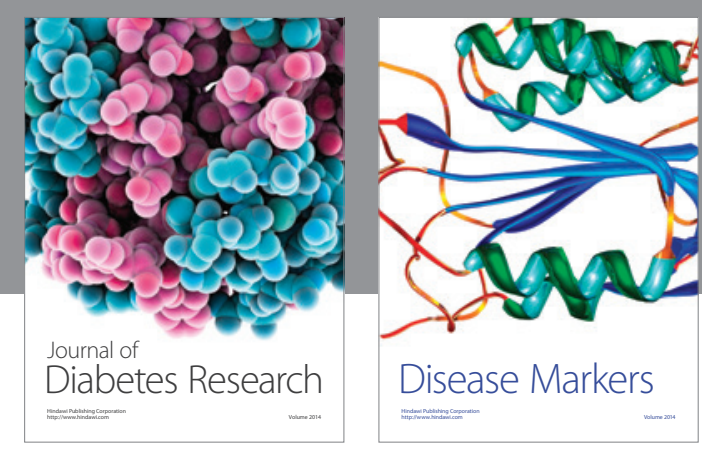

Disease Markers
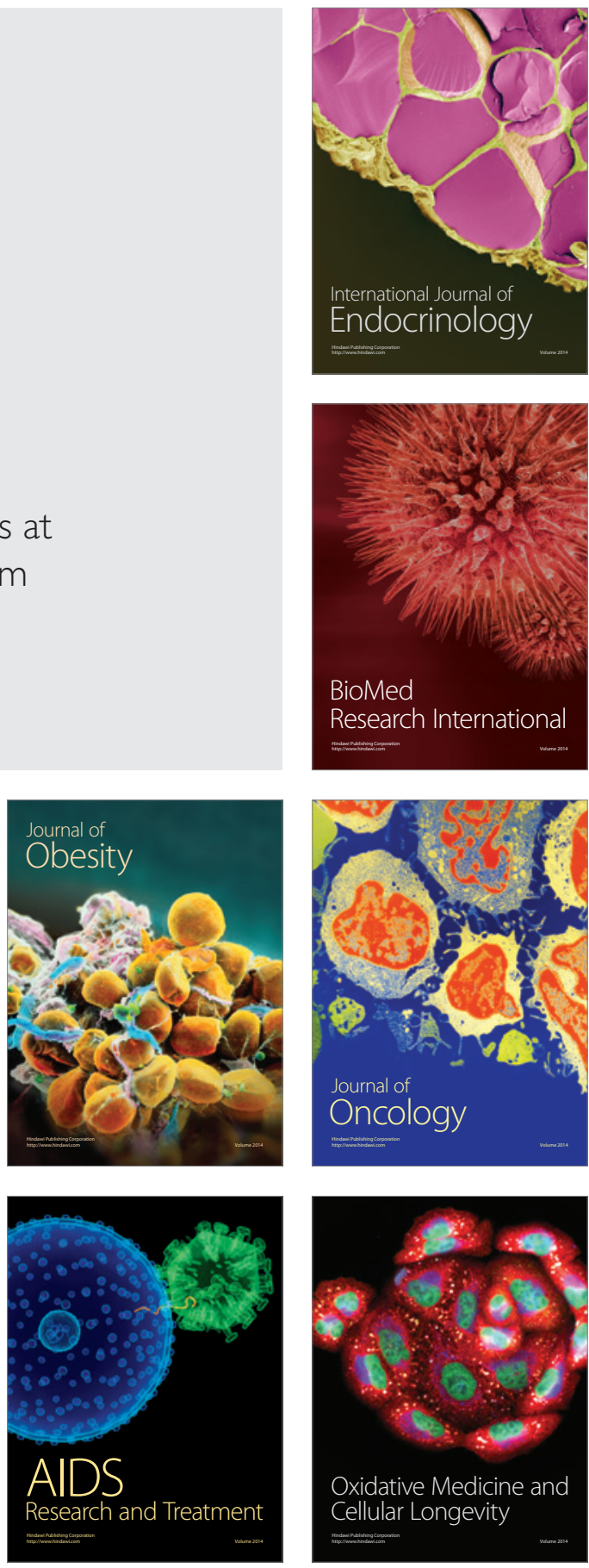